\title{
Efficacy of regorafenib combined with PD-1 inhibitors in elderly patients with advanced metastatic colorectal cancer
}

\section{Xiaobing Chen ( $\nabla$ zlyychenxb0807@zzu.edu.cn )}

Affiliated Cancer Hospital of Zhengzhou University, Henan Cancer Hospital

\section{Beibei Chen}

Affiliated Cancer Hospital of Zhengzhou University, Henan Cancer Hospital

Huichen Zhao

Affiliated Cancer Hospital of Zhengzhou University, Henan Cancer Hospital

Jinxi Huang

Affiliated Cancer Hospital of Zhengzhou University, Henan Cancer Hospital

\section{Huifang Lv}

Affiliated Cancer Hospital of Zhengzhou University, Henan Cancer Hospital

\section{Weifeng Xu}

Affiliated Cancer Hospital of Zhengzhou University, Henan Cancer Hospital

\section{Caiyun Nie}

Affiliated Cancer Hospital of Zhengzhou University, Henan Cancer Hospital

Jianzheng Wang

Affiliated Cancer Hospital of Zhengzhou University, Henan Cancer Hospital

Jing Zhao

Affiliated Cancer Hospital of Zhengzhou University, Henan Cancer Hospital

Yunduan He

Affiliated Cancer Hospital of Zhengzhou University, Henan Cancer Hospital

\section{Saiqi Wang}

Affiliated Cancer Hospital of Zhengzhou University, Henan Cancer Hospital

\section{Research Article}

Keywords: colorectal cancer, elderly patients, immunotherapy, programmed cell death protein 1 , regorafenib

Posted Date: February 28th, 2022

DOI: https://doi.org/10.21203/rs.3.rs-1271837/v1 
License: (c) (i) This work is licensed under a Creative Commons Attribution 4.0 International License. Read Full License 


\section{Abstract}

Objective: This is the first trial that wants to investigate the treatment patterns, clinical outcomes and prognostic factors of regorafenib plus PD-1 inhibitors therapy to Chinese elderly patients with advanced colorectal cancer.

Methods: A cohort of metastatic colorectal cancer patients with 60 years or older received treatment of regorafenib combined with PD-1 inhibitors was included in our analysis. The endpoints included overall survival (OS), progression free survival (PFS), and prognostic factors.

Results: In total, 24 patients were enrolled. The median age was 68 years; $62.5 \%$ were female. The median OS and PFS were 15.03 months (95\% Cl 7.0-23.0) and 4.0 months (95\% Cl 1.8-6.2), respectively. The objective response rate was $8.3 \%$ and disease control rate was $70.8 \%$. When compared with PFS and OS in different initial daily doses group of regorafenib (such as 40,80, 120 or $160 \mathrm{mg}$ ), we concluded that there were no significantly difference between these groups. And similar results were found in final daily doses groups. However, in terms of final daily doses, it showed a trend toward better PFS in $>80 \mathrm{mg}$ group compared to $\leq 80 \mathrm{mg}$ group (median PFS was 10.0 months in $>80 \mathrm{mg}$ group versus 3.5 months in $\leq 80 \mathrm{mg}$ group) . Patients with previous treated with regorafenib had longer median PFS than those without it (6.3 versus 2.8 months).

Conclusions: This real-world evidence confirms that Chinese elderly patients with advanced colorectal cancer may benefit from treatment of regorafenib combined with PD-1 inhibitors, similarly with this combination therapy strategies in all age patients.

\section{Introduction}

Colorectal cancer (CRC), as one of the most prevalent types of cancer in China, is still a leading cause of cancer related mortality ${ }^{[1]}$. Based on a multicenter retrospective study from China, the median age of metastatic CRC (mCRC) cases at diagnosis was 58 years, and almost half mCRC patients were age 60 year or older ${ }^{[2]}$. Therapeutic decisions involving elderly patients are a serious issue in oncology, because this group is characterized by a higher incidence of significant co-morbidities (cardiovascular disorders, metabolic disorders and liver disorders), decreased regenerative capacity of bone marrow (higher incidence and intensity of hematological complications of chemotherapy) as well as worse general performance ${ }^{[3]}$. Making all anticancer drugs available to elderly patients with $\mathrm{mCRC}$ is important to achieve the maximal benefit for long-term survival and maintain their quality of life.

Regorafenib, an oral small-molecule multikinase inhibitor, has demonstrated significantly improved survival in two randomized, double-blind, placebo-controlled phase 3 trials (CORRECT and CONCUR) ${ }^{[4,5]}$. Therefore, it is recommended as a standard third- or later-line therapy for refractory metastatic colorectal cancer (mCRC) by Chinese Society of Clinical Oncology (CSCO) guideline and other international guidelines ${ }^{[6-8]}$. Immune checkpoint inhibitors have showed promising therapeutic outcomes in advanced 
colorectal cancer with bearing mismatch repair-deficiency/microsatellite instability-high (dMMR/MSI-H)

tumors in recent years ${ }^{[7-10]}$. However, patients with mismatch repair-proficient/microsatellite stable (pMMR/MSS) colorectal cancer, who account for $95 \%$ of advanced colorectal cancer, could not benefit from this approach ${ }^{[11]}$.

Recent data suggest a possible synergic effect between regorafenib and immune checkpoint inhibitors, as has been shown in REGONIVO trial, which reported a response rate of $33 \%$ and median progressionfree survival of up to 7.9 months in a cohort of 24 Japanese patients with MSS treatment-refractory metastatic colorectal cancers ${ }^{[12]}$. In REGOTORI trial, which produced the similar results, the objective response rate (ORR) was $15.2 \%$ and median overall survival was 15.5 months in patients with refractory PMMR/MSS mCRC ${ }^{[13]}$. However, further research should be conducted to address this therapeutic strategy applying on elderly Chinese patients. This study aimed to investigate the treatment patterns, clinical outcomes and prognostic factors of regorafenib plus PD-1 inhibitors therapy to Chinese elderly patients with advanced colorectal cancer.

\section{Materials And Methods}

\section{Study population}

We retrospectively reviewed the patients treated with regorafenib plus PD-1 inhibitors for unresectable mCRC in Henan Cancer Hospital (China) from January 2019 to January 2021.

Patients were eligible for participation if they were 60 years of age or older and had histologically or cytologically confirmed mCRC. Patients were required to have failed to respond to all the available systemic agents, including fluoropyrimidine, oxaliplatin, irinotecan, bevacizumab, and cetuximab when applicable; and received at least one cycle of regorafenib plus PD-1 inhibitors. Besides, the medical record should be complete and legible. Patients who met any of the following criteria at the time of screening

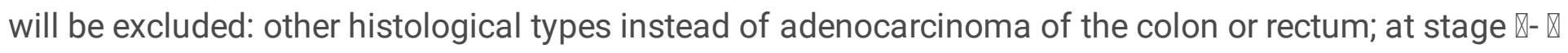
according to TNM staging system; Eastern Cooperative Oncology Group (ECOG) performance status of 3 or more. This work was approved by the ethics committee of Henan Cancer Hospital (Approval number: 2020103005). Patients were followed up by telephone questionnaires every three months until death or trial data cutoff date (July 31, 2021).

\section{Treatment and evaluation of therapeutic efficacy}

Regorafenib was prescribed at 40,80, 120 or $160 \mathrm{mg}$ daily from day 1 to day 21 of each 28 -day cycle. Depending on the patient's tolerability, the daily dose was allowed at the discretion of treating physicians. All patients received immune checkpoint inhibitors at the first day of regorafenib treatment, according to recommended dose: nivolumab 240 mg every 2 weeks, camrelizumab 200 mg every 2 weeks, sintilimab 200 mg every 3weeks, toripalimab 240 mg every 3 weeks, pembrolizumab 200 mg every 3weeks, tislelizumab 200 mg every 3weeks. 
Demographics, disease features and therapy information of patients were documented at baseline examinations. Tumour response was evaluated every 2 or 3-cycle treatment of PD-1 inhibitors, according to the Response Evaluation Criteria in Solid Tumour (RECIST) Version 1.1.

The primary endpoint was overall survival (OS), which was defined as the duration from treatment to death as a result of any cause. Other endpoints included progression-free survival (PFS), disease-control rate (DCR), objective response rate (ORR). Progression-free survival (PFS) was defined as the duration from treatment to first documented disease progression, or death. The objective response rate (ORR) was defined as complete response (CR) + partial response (PR)and the disease-control rate (DCR) was defined as $\mathrm{CR}+\mathrm{PR}+$ stable disease (SD). Adverse Events were graded and analysed according to National Cancer Institute Common Terminology Criteria for Adverse Events (NCI-CTCAE), Version 4.03.

\section{Statistical analysis}

All statistical analysis was performed using IBM SPSS Statistics for Windows Version 25.0 (IBM Corp., Armonk, New York, USA). Quantitative data were displayed as mean with standard deviation (SD), and qualitative data were expressed as number with percentage [No. (\%)]. Survival data were analysed using the Kaplan-Meier method and compared by log-rank test. $P$ value $<0.05$ was considered as statistically significant.

\section{Results}

\section{Study participants' clinical characteristics}

At a median follow-up of 16.2 months, a total of 24 patients were enrolled. 9 of these patients (37.5\%) were male; Median age was 68.0 years. The primary tumour site was the right-side colon in $33.3 \%(n=8)$ of patients, the left-side colon in $29.2 \%(n=7)$ and the rectum in $37.5 \%(n=9)$. The main metastatic sites were the liver and/or lung (91.7\%), only lung (25.0\%), only liver (29.2\%), distal lymph nodes (45.8\%) and peritoneum (16.7\%). Other baseline characteristics of the patients are presented in Table 1. 
Table 1

Baseline demographic and clinical characteristics of $24 \mathrm{mCRC}$ patients.

\section{Characteristics}

Age (year)

Median (range)

Gender

Male

Female

ECOG performance status

0

1

2

Primary tumor location

Colon

Right-side

Left-side

Rectum

Whether the primary tumor is resected

Resected

Not resected

Type of metastasis

With liver or lung metastasis

With only liver metastasis

With only lung metastasis

With liver and lung metastasis

With bone metastasis

With brain metastasis

With distant lymph nodes metastasis
68

$\mathrm{N}=24$ patients $\mathrm{n}(\%)$

$9(37.5 \%)$

$15(62.5 \%)$

$5(20.8 \%)$

$16(66.7 \%)$

$3(12.5 \%)$

$15(62.5 \%)$

$8(33.3 \%)$

$7(29.2 \%)$

$9(37.5 \%)$

$3(12.5 \%)$

$22(91.7 \%)$

$6(25.0 \%)$

$7(29.2 \%)$

$9(37.5 \%)$

$3(12.5 \%)$

$1(4.2 \%)$

$11(45.8 \%)$

Note: Abbreviations: $m C R C$, metastatic colorectal cancer; pMMR, mismatch repair-proficient; MSS, microsatellite stable; dMMR, mismatch repair-deficiency; MSI-H, microsatellite instability-high. 


\section{Characteristics}

With peritoneum metastasis

With other organs metastasis

Previous lines of chemotherapy

Two lines

Three or more lines

Previous targeted therapy

Bevacizumab

Cetuximab

With Bevacizumab and Cetuximab

Without previous targeted therapy

Combination immunization agents

Sintilimab

Carrelizumab

Nivolumab

Toripalimab

Pembrolizumab

Tislelizumab

Whether previous exposure to regorafenib

Exposure to regorafenib

No exposure to regorafenib

Whether with the local treatment

With the local treatment

Without the local treatment

Gene mutation status

RAS and BRAF wild-type

K-RAS mutant
$\mathrm{N}=24$ patients $\mathrm{n}(\%)$

$4(16.7 \%)$

$2(8.3 \%)$

$13(54.2 \%)$

$11(45.8 \%)$

$15(62.5 \%)$

$3(12.5 \%)$

$3(12.5 \%)$

$6(25.0 \%)$

$12(50.0 \%)$

$6(25.0 \%)$

$2(8.3 \%)$

$2(8.3 \%)$

$1(4.2 \%)$

$1(4.2 \%)$

8 (33.3\%)

$16(66.7 \%)$

$3(12.5 \%)$

$21(87.5 \%)$

$5(20.8 \%)$

$16(66.7 \%)$

Note: Abbreviations: mCRC, metastatic colorectal cancer; pMMR, mismatch repair-proficient; MSS, microsatellite stable; dMMR, mismatch repair-deficiency; MSI-H, microsatellite instability-high. 


\section{Characteristics}

BRAF mutant

Unknow

MMR or MSI status

pMMR or MSS

dMMR or MSI-H

Unknown

Note: Abbreviations: mCRC, metastatic colorectal cancer; pMMR, mismatch repair-proficient; MSS, microsatellite stable; dMMR, mismatch repair-deficiency; MSI-H, microsatellite instability-high.

Regorafenib plus PD-1 inhibitors were given to 13 patients (54.2\%) in third-line treatment, and 11 patients (45.8\%) in fourth-line treatment and beyond. The most common PD-1 inhibitors was Sintilimab (50.0\%), followed by carrelizumab (25.0\%), nivolumab (8.3\%), toripalimab 2 (8.3\%), pembrolizumab (4.2\%) and tislelizumab (4.2\%). There were 8 patients (33.3\%) previously received regorafenib therapy and no one having taken PD-1 inhibitors before entering this trial. MSI/MMR status data were available from 20 patients (83.3\%). Of these, only one patient (4.2\%) had an MSI-high tumor, and the remaining 19 patients (79.2\%) were MSS or MMR proficient.

\section{Primary efficacy}

The median OS and median PFS were 15.0 months( $95 \% \mathrm{Cl}, 7.0-23.0)$ and 4.0 months $(95 \% \mathrm{Cl}, 1.8-6.2)$, respectively (Fig. 1A\&B). Overall, the ORR and DCR were $8.3 \%$ and $70.8 \%$, respectively. CR, PR, SD, PD and patients without tumor response assessment were observed in 0, 2(8.3\%), $15(62.5 \%), 4(16.7 \%), 3$ (12.5\%) patients, respectively.

Specifically, patients with previous treated with regorafenib had longer median PFS than those without it. Median PFS was 6.3 months $(95 \% \mathrm{Cl}, 5.6-7.0)$ in the former and 2.8 months $(95 \% \mathrm{Cl}, 1.9-3.7)$ in the latter $(P=0.445$, Fig. $1 C)$. No significant associations for PFS could be seen in whether previously treated with bevacizumab $(P=0.874)$, K-RAS status $(P=0.150)$, tumor sites $(P=0.321)$ and any kind of PD- 1 inhibitors $(P=0.477)$.

$16.6 \%$ of patients $(n=4)$ started regorafenib at daily doses of $120 \mathrm{mg}, 79.2 \%$ of patients $(n=19)$ started at $80 \mathrm{mg}$ and $4.2 \%$ of patients $(n=1)$ started at $40 \mathrm{mg}$. There were $12.5 \%, 70.8 \%, 4.2 \%$ and $12.5 \%$ of patients received the final daily doses of $40,80,120$ and $160 \mathrm{mg}$, respectively. Dose modifications were performed in overall 9 patients (37.5\%), including dose reduction in 5 patients (20.8\%) and dose escalation in 4 patients (16.7\%). When compared with PFS and OS in high initial daily doses group (120 and $160 \mathrm{mg}$ ) and low initial daily doses group (40 and $80 \mathrm{mg}$ ), we concluded that there were no significantly difference between the two groups (median PFS, $P=0.643$; median OS, $P=0.279$ ). However, median PFS and OS in final high daily doses group (>80 mg) had better trends than those in final low 
daily doses group ( $\leq 80 \mathrm{mg}$ ). Median PFS was increased in final high daily doses group (10.0 months) vs (3.5 months) with final low daily doses group (Fig. 1D). Median OS was also increased in final $>80 \mathrm{mg}$ group (NR) versus in final $\leq 80 \mathrm{mg}$ group (15.0 months).

\section{Safety}

5 patients had a dose reduction due to adverse events, hand-foot skin reaction in 3 patients, hypertension in 1 patient, proteinuria in 1 patient. The most common adverse events were hand-foot skin reaction, fatigue, hypertension, diarrhea. The grade 3 to 4 adverse events occurred in 5 patients (20.8\%). The most common grade 3 to 4 events were hand-foot skin reaction, hypertension and oral mucositis.

\section{Discussion}

As the majority of CRC cases display a molecular MSS/pMMR profile, it is particularly meaningful to investigate the clinical applications of adaptive immune or combination regimens in MSS CRC patients. Several studies have shown that the combination regimen using regorafenib combined with PD-1 inhibitors has promising efficacy for those patients ${ }^{[12-18]}$. The highest ORR which can be seen in REGONIVO study, was 33\%; and the median OS from REGOTORI was up to 15.5 months. However, there is a lack of clear evidence regarding real world effects of this therapeutic strategy, especially in elderly Chinese patients.

In this single-center, retrospective study involving Chinese elderly patients with refractory advanced colorectal cancer, the median age of patients enrolled in our study is 68 years, which is older than that in

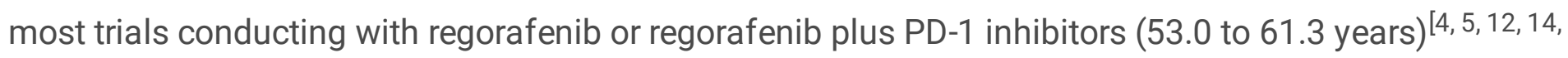
15]. Notably, the reported median OS of 15.03 months was comparable to those reported in REGOTORI (15.5 months) ${ }^{[13]}$. The combination using regorafenib plus PD-1 inhibitors seemed to achieve a better OS and ORR (8.3\%) than regorafenib alone (ORR 1-4\%; OS 6.4-8.8 months) for patients with $\mathrm{mCRC}^{[4,5]}$. Although the combination regimen yields a response rate of less than $10 \%$, which lower than those reported in REGOTORI (15.2\%) ${ }^{[13]}$ and REGONIVO $(36.0 \%)^{[12]}$, more than $70 \%$ of patients $(70.8 \%)$ in our study achieved disease control, which was superior to the results of single-agent regorafenib, and similar to those from another China trial ${ }^{[4,5,14]}$. Hence, there is a possible reason that patients achieved higher DCR resulted in better OS in our trial. Overall, these results showed that it was worth to be recommended for regorafenib plus PD-1 inhibitors for elderly patients with $\mathrm{mCRC}$, especially in patients with a molecular MSS/pMMR profile.

Further analyses of effectiveness in our study showed that there were no obvious correlation for survival between patients only had liver metastasis and lung metastasis $(P>0.05)$. In addition, PFS were similar for patients with KRAS wild-type versus mutant, left-versus right-sided tumours, and among various types of PD-1 inhibitors. However, patients with previous treated with regorafenib had longer PFS than those without it ( 6.30 months versus 2.80 months, $P>0.05)$. Several reports showed that regorafenib modulated immuno-suppressive tumor microenvironment by blocking VEGFRs, TIE2, and CSF-1R, 
RET/Src axis signal pathways, and increased intratumoral CD 45 + leukocytes, $C D 8^{+} \mathrm{T}$ cells, which would enhance anti-tumor immunity when using regorafenib alone and plus various immunotherapies ${ }^{[19-21]}$. This could be why patients who had previous received with regorafenib obtained longer PFS. Further resources and research should be conducted to address time of administration for regorafenib plus PD-1 inhibitors.

We noticed that most patients in our cohort started at the lower dose of regorafenib. All were at the doses of less than $120 \mathrm{mg}$ and patients started at the dose of $\leq 80 \mathrm{mg}$ accounted for $83.4 \%$. During follow-up, 4 patients received higher dose and 5 patients had dose reduction. Consequently, the number of patients received less than $80 \mathrm{mg}$ doses stayed the same with those in baseline; whereas 4 patient $(16.7 \%)$ raised the doses up to $120 \mathrm{mg}$ or $160 \mathrm{mg}$. When compared with PFS and OS in different initial daily doses group of regorafenib ( $\leq 80$ versus $>80 \mathrm{mg}$ ), we concluded that there were no significantly difference between these groups. And similar results were found in final daily doses groups. However, it showed a trend toward better PFS in patients with final daily doses $>80 \mathrm{mg}$ group compared to $\leq 80 \mathrm{mg}$ group (median PFS was 10.0 months in $>80 \mathrm{mg}$ group versus 3.5 months in $\leq 80 \mathrm{mg}$ ). Considering the safety and tolerability profile of regorafenib, we argue that it should be started with a lower dose for regorafenib and adjusted it until the maximum tolerable dose was reached, by periodic follow-up and communication in time between nurse and patients. This strategy could contribute to enhance the tolerability and improve adherence, and reduce the risk of adverse events from regorafenib and/or PD-1 inhibitors.

The limitations of our study include its retrospective nature, the inadequate data on toxicity evaluation, the small sample size, and the short median follow-up. Besides, there were 4 PD- 1 inhibitors just approved in China but no other countries, which means these therapeutic drugs not available to other countries. However, the results may provide a clearer picture of the efficacy and safety of regorafenib plus PD-1 inhibitors in elderly Chinese patients with mCRC.

To our knowledge, this is the first paper describing the detailed information of regorafenib plus PD-1 inhibitors in elderly Chinese patients with $\mathrm{MCRC}$ in real-world settings. This real-world evidence confirms that Chinese elderly patients with advanced colorectal cancer can benefit from treatment of regorafenib combined with PD-1 inhibitors, similarly with this combination therapy strategies in all age patients. However, further larger cohorts research should investigate whether the PFS advantage in high dose group could eventually lead to improved OS outcomes. Besides, considering the quality of life, closely monitoring and management for adverse drug events are needed.

\section{Declarations}

\section{Ethics approval and consent to participate}

This work was approved by the ethical committee of Henan Cancer Hospital (Approvalnumber:2020103005). All subjects signed their informed consents before treatments. All methods were performed in accordance with the relevant guidelines and regulations. 


\section{Consent for publication}

Not applicable

\section{Availability of data and materials}

The datasets presented in this article are not readily available because of Chinese regulations and conditions for informed consent. Requests to access the dataset should be directed to Beibei Chan, zlyychenbb1429@zzu.edu.cn.

\section{Competing interests}

Conflicts of Interest: The authors declare no conflict of interest.

\section{Funding}

This study was supported by the 1000 Talents Program of Central plains (No.204200510023 for Xiaobing Chen), Science and Technique Foundation of Henan Province (No.212102310771for Weifeng Xu, No.202102310121 for Jianzheng Wang) $₫$ Medical Science and Technique Foundation of Henan Province (No. LHGJ20210172 for Beibei Chen)

\section{Authors' contributions}

Beibei Chen is expected to have made substantial contributions to the conception and designed of the work, together drafted it. Huichen Zhao, Jinxi Huang, Huifang Lv, Weifeng Xu were responsible for data collection. Jing Zhao, Yunduan He, Saiqi Wang were responsible for statistical analysis and making tables and figures. Caiyun Nie, Jianzheng Wang revised the manuscript. Xiaobing Chen was in charge of the protocol and approved the submitted version. All authors had reviewed the manuscript.

\section{Acknowledgements}

We would like to give our thanks to Junyan Zhang (Bothwin Clinical Study Consultant) for English editing.

\section{Author information}

Beibei Chen ${ }^{1,3}$, Huichen Zhao ${ }^{1,3}$, Jinxi Huang ${ }^{2}$, Huifang $\mathrm{Lv}^{1,3}$, Weifeng $\mathrm{Xu}^{1,3}$, Caiyun $\mathrm{Nie}^{1,3}$, Jianzheng Wang $^{1,3}$, Jing Zhao ${ }^{1,3}$, Yunduan $\mathrm{He}^{1,3}$, Saiqi Wang ${ }^{1,3}$, Xiaobing Chen ${ }^{* 1,3}$ 
${ }^{1}$ Department of Medical Oncology, Affiliated Cancer Hospital of Zhengzhou University, Henan Cancer Hospital, Zhengzhou, Henan Province 450008, China. ${ }^{2}$ Department of Gastrointestinal Surgery, Affiliated Cancer Hospital of Zhengzhou University, Henan Cancer Hospital, Zhengzhou, Henan Province 450008, China. ${ }^{3}$ Zhengzhou Key Laboratory for Precision Therapy of Gastrointestinal Cancer, Zhengzhou, Henan Province 450008, China.

Correspondence to: Xiaobing Chen, Department of Medical Oncology, Affiliated Cancer Hospital of Zhengzhou University, Henan Cancer Hospital, Zhengzhou, Henan Province 450008, China, zlyychenxb0807@zzu.edu.cn, +8613937100233.

\section{References}

1. Zhang S, Sun K, Zheng R, Zeng H, Wang S, Chen R, Wei W, He J: Cancer incidence and mortality in China, 2015. Journal of the National Cancer Center2021, 1(1):2-11.

2. Xu R, Wang W, Zhu B, Lin X, Ma D, Zhu L, Zhao Q, Nie Y, Cai X, Li Qet al: Disease characteristics and treatment patterns of Chinese patients with metastatic colorectal cancer: a retrospective study using medical records from China. BMC Cancer2020, 20(1):131.

3. Stec R, Bodnar L, Smoter M, Mączewski M, Szczylik C: Metastatic colorectal cancer in the elderly: An overview of the systemic treatment modalities (Review). Oncol Lett2011, 2(1):3-11.

4. Grothey A, Cutsem EV, Sobrero A, Siena S, Falcone A, Ychou M, Humblet Y, Bouché O, Mineur L, Barone Cet al: Regorafenib monotherapy for previously treated metastatic colorectal cancer (CORRECT): an international, multicentre, randomised, placebo-controlled, phase 3 trial. The Lancet2013, 381(9863):303-312.

5. Li J, Qin S, Xu R, Yau TCC, Ma B, Pan H, Xu J, Bai Y, Chi Y, Wang Let al: Regorafenib plus best supportive care versus placebo plus best supportive care in Asian patients with previously treated metastatic colorectal cancer (CONCUR): a randomised, double-blind, placebo-controlled, phase 3 trial. The Lancet Oncology2015, 16(6):619-629.

6. Dong C, Ding Y, Weng S, Li G, Huang Y, Hu H, Zhang Z, Zhang S, Yuan Y: Update in version 2021 of CSCO guidelines for colorectal cancer from version 2020. Chinese Journal of Cancer Research2021, 33:302-307.

7. Chiorean E, Nandakumar G, Fadelu T, Temin S, Alarcon-Rozas A, Bejarano S, Croitoru A, Grover S, Lohar P, Odhiambo DAet al: Treatment of Patients With Late-Stage Colorectal Cancer: ASCO Resource-Stratified Guideline. JCO Global Oncology2020, 6:414-438.

8. Van Cutsem E, Cervantes A, Adam R, Sobrero A, Van Krieken JH, Aderka D, Aranda Aguilar E, Bardelli $A$, Benson A, Bodoky Get al: ESMO consensus guidelines for the management of patients with metastatic colorectal cancer. Ann Onco/2016, 27(8):1386-1422.

9. Diaz LA, Le DT: PD-1 Blockade in Tumors with Mismatch-Repair Deficiency. The New England journal of medicine2015, 373(20):1979. 
10. Overman MJ, McDermott R, Leach JL, Lonardi S, Lenz H-J, Morse MA, Desai J, Hill A, Axelson M, Moss RAet al: Nivolumab in patients with metastatic DNA mismatch repair-deficient or microsatellite instability-high colorectal cancer (CheckMate 142): an open-label, multicentre, phase 2 study. The Lancet Oncology2017, 18(9):1182-1191.

11. André T, Shiu K-K, Kim TW, Jensen BV, Jensen LH, Punt C, Smith D, Garcia-Carbonero R, Benavides M, Gibbs Pet al: Pembrolizumab in Microsatellite-Instability-High Advanced Colorectal Cancer. New England Journal of Medicine2020, 383(23):2207-2218.

12. Fukuoka S, Hara H, Takahashi N, Kojima T, Kawazoe A, Asayama M, Yoshii T, Kotani D, Tamura H, Mikamoto Yet al: Regorafenib Plus Nivolumab in Patients With Advanced Gastric or Colorectal Cancer: An Open-Label, Dose-Escalation, and Dose-Expansion Phase lb Trial (REGONIVO, EPOC1603). Journal of Clinical Oncology2020, 38:JC0.19.03296.

13. Wang F, He M-M, Yao Y-C, Zhao X, Wang Z-Q, Jin Y, Luo H-Y, Li J-B, Wang F-H, Qiu M-Zet al: Regorafenib plus toripalimab in patients with metastatic colorectal cancer: a phase lb/Il clinical trial and gut microbiome analysis. Cell Reports Medicine2021, 2(9):100383.

14. Cousin S, Cantarel C, Guegan J-P, Gomez-Roca C, Metges J-P, Adenis A, Pernot S, Bellera C, Kind M, Auzanneau Cet al: Regorafenib-Avelumab Combination in Patients with Microsatellite Stable Colorectal Cancer (REGOMUNE): A Single-arm, Open-label, Phase II Trial. Clinical Cancer Research2021, 27(8):2139.

15. Rui Liu XW, Zhi Ji, Ting Deng, Le Zhang, Yuchong Yang, Tao Ning, Ming Bai, Shaohua Ge, Hongli Li, Jingjing Duan, Yi Ba: A single-arm study on the efficacy and safety of regorafenib plus sintilimab as salvage-line treatments in non-MSI-H metastatic colorectal cancer. J Clin Oncol2021, 39(e15560).

16. Hangyu Zhang YZ, Zhou Tong, Lulu Liu, XuDdong Zhu, QiHan Fu, Xuanwen Bao, Xiaomeng Dai, Weijia Fang, Peng Zhao: Retrospective pilot study of regorafenib combined with ICls in the third-line treatment of advanced colorectal cancer. J Clin Onco/2021, 39(e15582).

17. Kaili Yang LH, YunBo Zhao, Yang Ge,Qin Li,Yusheng Wang,Shikai Wu,Yan Zhang,Chuanhua Zhao,Jing Zhou,Lin Zhao: Regorafenib plus PD-1 inhibitors in Chinese patients with microsatellite stable/mismatch repair proficient metastatic colorectal cancer: A real-world study. J Clin Onco/2021, 39(e15585).

18. Fakih Marwan KPR, David Z.Chang,Johanna C.Bendell,Timothy Larson,Allen L.Cohn,Timothy K.Huyck,David Cosgrove,Joseph A.Fiorillo,Lawrence E.Garbo,Shruthi Raximohan,Von Potter,David R.D'Adamo,Neelesh Sharma,Ying A.Wang,Sabine Coppieters,Matthias Herpers,Carolina Soares Viana de Oliveira,Andrew S.Paulson: Single-arm, phase 2 study of regorafenib plus nivolumab in patients with mismatch repair-proficient (pMMR)/microsatellite stable (MSS) colorectal cancer (CRC). J Clin Oncol2021, 39(3560).

19. Arai H, Battaglin F, Wang J, Lo JH, Soni S, Zhang W, Lenz H-J: Molecular insight of regorafenib treatment for colorectal cancer. Cancer Treatment Reviews2019, 81:101912.

20. Tsai AK, Khan AY, Worgo CE, Wang LL, Liang Y, Davila E: A Multikinase and DNA-PK Inhibitor Combination Immunomodulates Melanomas, Suppresses Tumor Progression, and Enhances 
Immunotherapies. Cancer Immunol Res2017, 5(9):790-803.

21. Wu R-Y, Kong P-F, Xia L-P, Huang Y, Li Z-L, Tang Y-Y, Chen Y-H, Li X, Senthilkumar R, Zhang H-Let al: Regorafenib Promotes Antitumor Immunity via Inhibiting PD-L1 and ID01 Expression in Melanoma. Clinical Cancer Research2019, 25(14):4530.

\section{Figures}

A

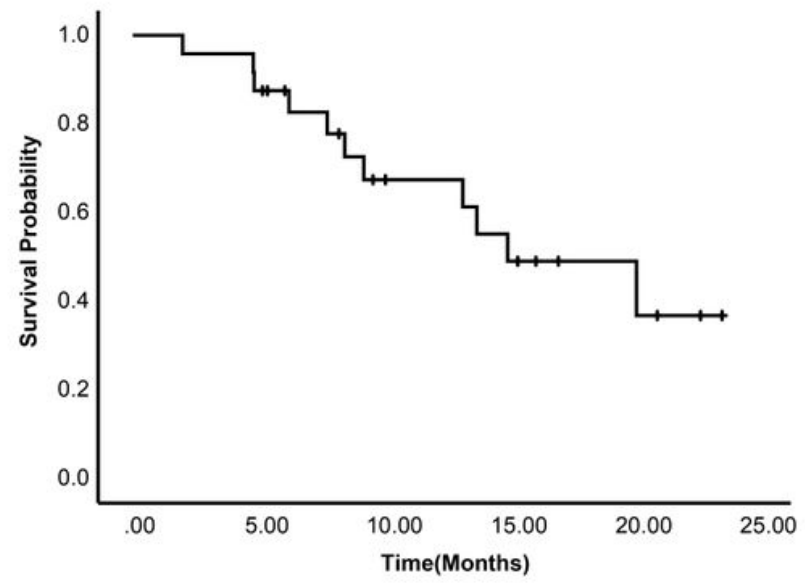

C

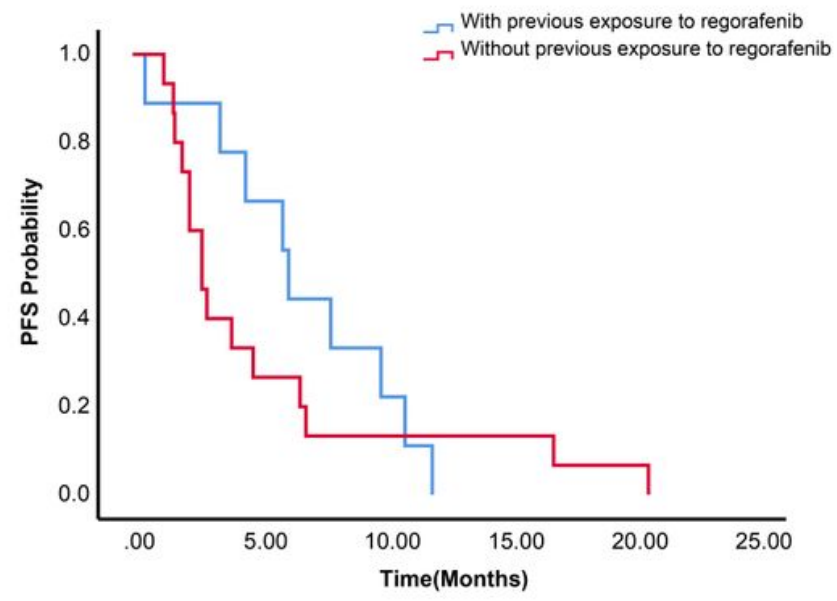

B

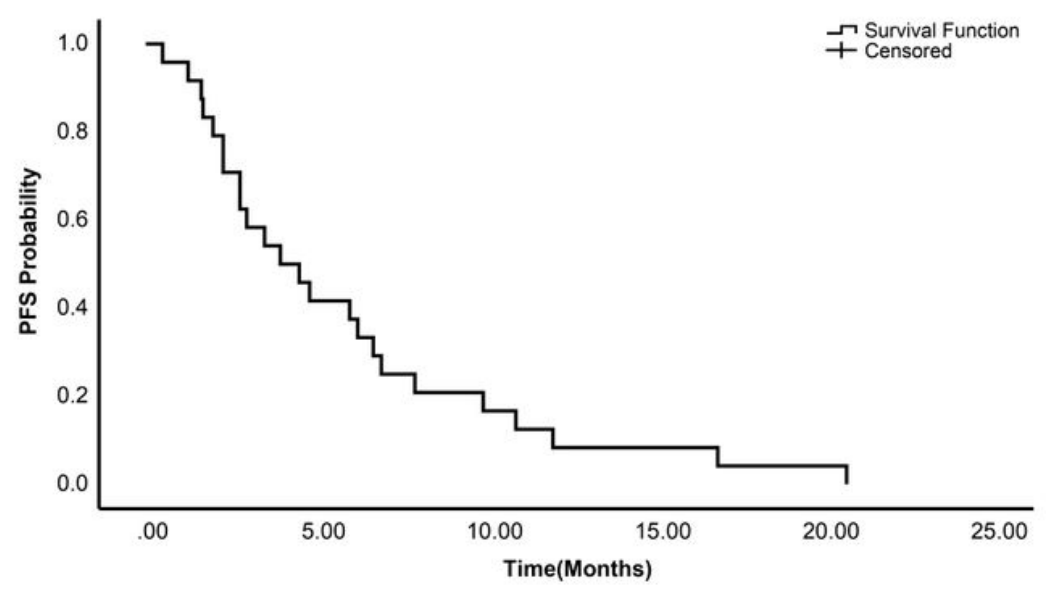

D

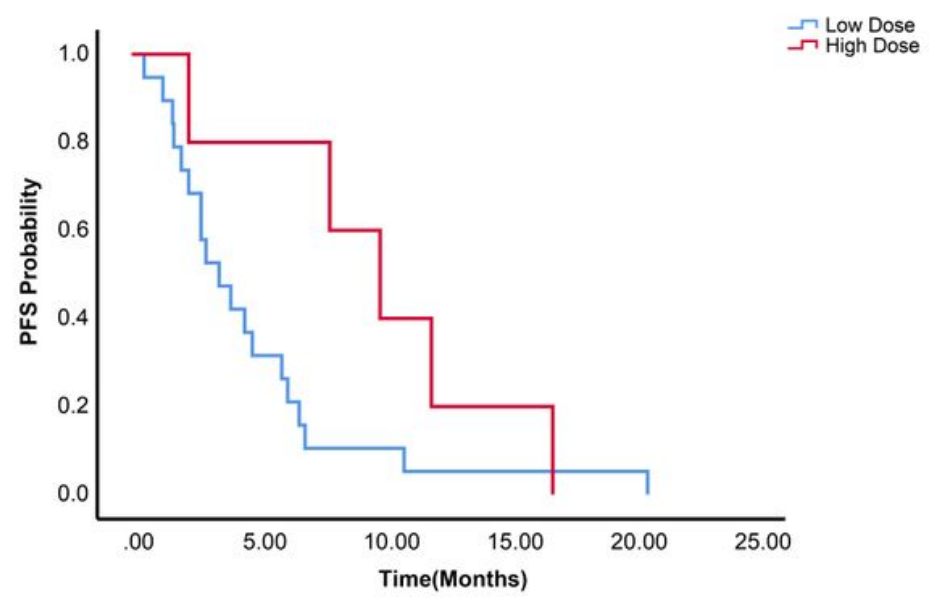

\section{Figure 1}

Kaplan-Meier survival curves. PFS of 21 evaluable patients (A). OS of the whole cohort (B). PFS for patients with or without previous exposure to regorafenib (C). PFS for patients with various final dose level (D). 